\section{Civilizar}

ISSN 1657-8953 e-ISSN: 2619-189X

\title{
La familia, primer ámbito de educación cívica*
}

The family: the First Area of Civic Education

Iván Darío Moreno Aceros

Pámela Leyva-Townsend

Ciro Parra Moreno"

"Artículo de reflexión.

$\S$ Licenciado en filosofía, Magíster en Educación y Doctorando en estudios Sociales de la Universidad Externado de Colombia. Actualmente es profesor-investigador del Instituto de la Familia de la Universidad de la Sabana.

Correo electrónico:

ivanma@unisabana.edu.co

(iD) $0000-0003-1308-6184$

Administradora de Instituciones de Servicios de la Universidad de La Sabana y Magíster en Estudios Sociales de la Universidad del Rosario. Actualmente es profesora del Instituto de La Familia de la Universidad de La Sabana en Chía, Colombia.

Correo electrónico:

pamela.leyva1@unisabana.edu.co

(iD) $0000-0002-9256-8271$

${ }^{\pi}$ Licenciado en administración y supervisión educativa de la Universidad de la Sabana y Doctor en Pedagogía de la Universidad de Navarra. Actualmente es Decano de la Facultad de Educación de la Universidad de la Sabana.

Correo electrónico:

ciro.parra@unisabana.edu.co

(iD) $0000-0001-8774-6531$

Como citar:

Moreno-Acero, I., Leyva-Townsend, P. y Parra-Moreno, C. (2019). La Familia, primer ámbito de educación cívica. Civilizar: Ciencias Sociales y Humanas, 19(37), 43-54. doi: 10.22518/usergioa/jour/ ccsh/2019.2/ao6

\section{Resumen}

Las circunstancias históricas y sociales actuales que evidencian un deterioro de la democracia y de la capacidad para actuar como ciudadanos críticos y reflexivos, exigen fortalecer tanto los ejercicios de formación ciudadana como el rol de los nuevos actores. En el caso del presente artículo se explora el rol de la familia en la educación cívica. El objetivo de este texto es mostrar que la familia es un ámbito educativo central en la formación de un conjunto de virtudes cívicas y, por ende, en el desarrollo de una capacidad ética de actuación política.

\section{Palabras Clave}

Familia, educación cívica, virtudes, actuación política, socialización política.

\section{Abstract}

The current historical and social circumstances, which evidence a deterioration of democracy and of the capacity to act as critical and reflexive citizens, demand the strengthening of both citizen training exercises and the role of new actors. In the present essay, the role of the family in civic education is explored. The text aims to demonstrate that the family is a central educational sphere in the formation of a set of civic virtues and, therefore, in the development of an ethical capacity for political action.

\section{Keywords}

Family, civic education, virtues, political behavior, political popularization. 


\section{Introducción}

La familia es reconocida como núcleo básico de la sociedad, como unidad ecosistémica clave, es decir, un espacio cotidiano en el que se entretejen redes de apoyo, confianza, mutualidad y solidaridad (Estupiñán y Hernández, 2007; Muaze, 2016). También se comprende como un ámbito para la vida en la medida en que permite la constitución del ser de la persona, de su identidad y de los rasgos que lo definen como un animal político y un ser social. De igual modo, como un agente social y educativo o un actor preponderante en los procesos de socialización de los principales códigos sociales, de los principios comunes que condicionan la existencia y el funcionamiento de una sociedad (Rodríguez, 2013; Plaza y Caro, 2016).

De esta forma, la familia participa activamente de las prácticas de socialización política, pues es ella quien acompaña de manera permanente el proceso de agenciamiento y desarrollo que se inicia con el nacimiento. La formación inicial configura de modo ininterrumpido y definitivo nuestra identidad, y empieza en la unidad familiar cotidiana; allí, cada uno de los espacios incidirá en la formación y en la configuración de un sentido amplio o restringido de alteridad, comunidad, bien, etc.

De acuerdo con Osorio (2004), hay diversas formas de socialización política: formal, planificada e informal. Para el contexto familiar, se pueden presentar cualquiera de estas, o las tres; la variabilidad depende del interés y de la intención que cada familia tenga con respecto al proceso formativo y al tipo de enseñanza y sociedad que quiera construir. A esto dice:

Podemos realizar una distinción entre socialización formal, planificada y prescrita por las instituciones sociales, e informal, más propia de las vivencias y experiencias de la vida cotidiana. La socialización ha de ser entendida en el marco de un proceso dinámico que genera un intercambio complejo y a través del cual cada sujeto interactúa con su medio y con agentes de muy diverso tipo. (p. 1).

Las tensiones y transacciones de la cotidianidad familiar, así como las prácticas educativas intencionadas o no, tienen un alcance en la formación y estructuración de la identidad política de las personas que conforman la familia. Los procesos transaccionales de intercambio y comunicación de códigos sociales llevan a que la socialización política no sea un proceso unidireccional, es decir, de adultos a menores, sino uno multidireccional; las reflexiones que comparten los niños sobre su realidad podrían incidir en la transformación del horizonte de comprensión de sus familiares y, por tanto, en el de sus principios. En consecuencia, reducir la socialización política a un ejercicio unidireccional, supondría el desconocimiento de los niños, en primer lugar, como sujetos sociales de derecho y, en segundo lugar, como agentes sociales, cuya voz y participación que, de acuerdo con Zuluaga (2004), es necesaria para la estructuración de la comunidad.

Así las cosas, el propósito de este ensayo es ofrecer una perspectiva reflexiva sobre el rol que la familia cumple en la educación cívica, a través de sus prácticas cotidianas de socialización política; perspectiva orientada a demostrar que la familia es un ámbito crucial para el desarrollo de un conjunto de virtudes cívicas y, por ende, para la formación de un tipo de capacidad virtuosa de actuación política.

\section{Familia}

La familia es una unidad ecosistémica de vida que se encarga, en un sentido extenso, del proceso de formación de los sujetos. "La familia es una comunidad de amor y solidaridad" (D'Agostino, 1991, p. 18). Es una unidad ecosistémica de vida porque en su intimidad relacional y en su vida ordinaria, se producen espacios vitales por medio de los que se constituye la persona. De esta manera, desde el momento mismo de la gestación, es un actor decisivo y clave en la constitución y configuración de las cualidades o características que definen al hombre como persona.

En efecto, como unidad ecosistémica es una fuente formativa primaria cuya labor es proteger, cuidar y formar, así como humanizar y humanizar. Esto implica que, mediante las prácticas cotidianas de crianza, sensibiliza y concientiza a la persona sobre su condición humana, de ser racional, libre y social, que puede participar activamente en la configuración y dotación de sentido del mundo social y natural que le rodea.

Entenderla como unidad ecosistémica conlleva a aceptarla como un organismo vivo, que se adapta a los tiempos y espacios; como una célula social interdependiente que es afectada y afectante del medio que habita. Ella posee voluntad, libertad y capacidad para incidir de forma activa en la construcción de la realidad, tanto de su medio social como en su intimidad. La vida ordinaria de la familia, con 
sus situaciones interactivas conflictivas, dialógicas, emocionales y normativas, permite la transmisión de los principales esquemas de pensamiento y acción que se requiere para vivir en comunidad y, desplegar con amplitud los rasgos humanos, es decir, cada una de las dimensiones que definen la personalidad.

En la perspectiva de familia que propone Estupiñán y Hernández (2007) se halla que:

La familia es una unidad ecosistémica de supervivencia y de construcción de solidaridades de destino, a través de los rituales cotidianos, los mitos y las ideas acerca de la vida, en el interjuego de los ciclos evolutivos de todos los miembros de la familia en su contexto sociocultural... La familia es una forma de vida en común, constituida para satisfacer las necesidades emocionales de los miembros a través de la interacción. El afecto, el odio, la complacencia y todos los demás sentimientos, muchas veces encontrados, proveen un ambiente dentro del cual los individuos viven procesos que determinarán su estilo de interacción en otros contextos. (pp. 57-61).

Lo anterior implica que la familia no es una institución pasiva o acrítica que se deja llevar por las circunstancias históricas, políticas y sociales; también puede incidir en el curso de los eventos, ya que está en capacidad para elegir la forma en que educa, los sentidos que le otorga a los principios que transmite y los vehículos que emplea para este fin. Es un espacio de supervivencia endógeno y exógeno.

Como espacio endógeno de supervivencia facilita la conservación de la vida de sus miembros y, como espacio exógeno de supervivencia garantiza la conservación y la promoción de la vida en los medios sociales y naturales. En el primer caso, a través del amor, de la promoción permanente de la dignidad inherente que posee la vida, del respeto constante de los derechos humanos de sus miembros y de la defensa incansable de la vida ante las circunstancias límite en que su valor se torna relativo; esta institución es conservadora y promotora de la vida. En el segundo caso, implica que sus prácticas se orientan al desarrollo de un sentido moral del compromiso que la persona posee para sí y para con "otros" (Hall, 2016).

Además, es una red de solidaridades de destino que por medio de los encuentros cotidianos afianza los lazos de unidad, intimidad y mutualidad o apoyo. Cada uno de estos elementos brinda la seguridad y la confianza de que se pertenece a un lugar; que se poseen un espacio físico y simbólico de origen y de retorno.

Esta red de apoyo es una estructura social desde la que se acompaña el crecimiento humano, cuyo propósito es brindar un soporte espiritual-intelectual, material-formal y emocional-sentimental a través de la vinculación colaborativa. Como red de apoyo, facilita el intercambio de saberes, la construcción contributiva de lo familiar y la articulación de las necesidades, expectativas y creencias alrededor de los fines comunes que ella y sus miembros se han planteado de manera singular.

Los espacios familiares son lugares transaccionales donde se intercambian y comunican, de manera educativa, las formas en que cada miembro de la familia habita, experimenta y comprende el mundo. De esta forma, la familia también es un espacio dialogal-colaborativo que propicia la construcción de un sentido de mundo común. Este principio determina la forma en que se socializan las creencias o representaciones de los fenómenos particulares y su totalidad, ya que las prácticas educativas familiares no pueden ser un proceso de imposición o de entera apertura desestructurada, sino un diálogo equilibrado-democrático intergeneracional que facilite la adaptación, el aprendizaje y el tránsito de la persona por el mundo.

Como espacio dialógico dinámico evoluciona conforme cambian sus miembros en el proceso de ampliación de sus horizontes de comprensión. No obstante, esta evolución o adaptación debe ser tendiente al mantenimiento de sus funciones sustantivas, al fortalecimiento de su capacidad formativa y su rol social como una institución estructuradora de la realidad.

Verla como una institución natural que se adapta a los retos y vicisitudes equivale a advertirla como una institución perenne, cuyas funciones y tareas son irremplazables. De esta forma, la familia "evoluciona gracias a las perturbaciones que surgen de su interior y de su entorno y se reorganizan permanentemente en virtud de sus propios mecanismos redundantes, inherentes a su naturaleza" (Estupiñán y Hernández, 2007, p. 65).

En este sentido, y siguiendo a Estupiñán y Hernández, es un sistema vivo que desarrolla fortalezas y capacidades "para protegerse de los eventos inesperados y para promover la adaptación después de las crisis" (2007, p. 65). Esta idea de familia como 
una institución que se puede reparar y recobrar en los momentos de crisis, lleva también a entender que:

La familia es un bien de la humanidad que coevoluciona con todos los demás sistemas sociales, participa como unidad activa en el interjuego social y requiere en consecuencia respecto a su autonomía y reconocimiento de su pleno protagonismo en la conservación constructiva de los individuos y de la sociedad... La familia no es solo objeto de derechos y de aplicación de políticas que la protejan. Debe ser considerada un actor social participante en la construcción de las políticas sociales que la afectan. (pp. 6-19).

Por tanto, a la familia se le debe entender como un actor social clave, un bien en sí mismo, un organismo vivo; una célula cuya función principal consiste en constituir la sociedad por medio de la formación, cuidado y protección de la persona. Las características que la definen como un actor social clave, y no como un elemento subsidiario y secundario de la sociedad, están relacionadas con los roles sociales, políticos, culturales e históricos que desempeña; o lo que es igual, con las acciones sociales sustantivas tendientes al mantenimiento y prolongación de sociedades justas, responsables, equitativas y democráticas (Nora, 2011). Se ha insistido en que aquellas cuestiones vienen unidas a su labor cotidiana formativa, implícita o explícita, dirigida a que el ser humano se haga persona y participe activamente en la construcción de lo común.

\section{Educación familiar}

Esta es una institución que le dona a los sujetos y a la sociedad un conjunto de esquemas que condicionan la acción, de principios normativos que regularán sus prácticas sociales privadas y públicas. Desde la antropología, la sociología y la filosofía se ha estudiado y promovido este valor. Por ejemplo, para Donati (2003) la familia es la raíz de la sociedad, por lo que, si ella "se rompe, también la sociedad se rompe; si la familia se vuelve líquida, también la sociedad se vuelve líquida" (p. 15). Por otra parte, para Schrecker (1970) "sirve de apoyo a la civilización y, en cierto modo, es apoyada por esta... es, incuestionablemente, un elemento intrínseco de la vida humana" (p. 275). Atendiendo a otro referente, en la Declaración Universal de los Derechos Humanos se consagra la familia como elemento natural $y$ fundamental de la sociedad (Art. 16).

Desde la perspectiva de Estupiñán y Hernández:
La familia le aporta a la vida social un matiz especial como espacio de la subjetividad, sitio de una interioridad libre y desenvuelta donde tiene lugar el cultivo de la identidad del sujeto. La familia sienta las bases de su desarrollo personal y aporta la protección afectiva. La familia es la mediadora entre lo público y lo privado gracias a la formación que requiere el ejercicio de la ciudadanía. La familia expresa una forma particular de la vida social, pues por ella atraviesa la construcción de lo público, el retraimiento a lo privado y el disfrute de lo íntimo. La familia es un actor decisorio en el desarrollo social, una instancia deliberante y actuante de la sociedad civil. (p. 17).

Como se puede comprender desde los diferentes aportes mencionados, la familia no se agota en la privacidad del hogar, por el contrario, cada una de sus rutinas impacta en la sociedad. Es la garante de socialización inicial de los valores y normas que una comunidad de vida posee. Por ello, sobre la familia recae la transmisión de la cultura y los principales símbolos que constituyen los sentidos de persona y mundo, con los que un sujeto se relaciona y desenvuelve socialmente (Valdés, Carlos, Quintana y Madrid, 2016).

Como un espacio de relación y socialización, es un escenario que, de modo intencionado o no, genera un conjunto de prácticas sociales con un alcance educativo en el desarrollo y formación de los niños y jóvenes (Matos, 2015). Es un lugar que no está exento de tensiones (conflictos-choques-disyuntivas) entre ella y la sociedad, entre padres e hijos, y entre los hijos y la sociedad.

Las prácticas educativas que cada familia emplea varían de una cultura a otra, lo que no varía es el fin con el que una familia trasmite los principios sociales, ya que, estos le facilitan al niño participar activamente en la comunidad de vida de sus padres. Siguiendo el planteamiento de que la familia es escenario que cumple una labor educativa sustantiva, Donati (2003) propone dos ideas al respecto: La primera, ella es el origen de la sociedad humana. Al respecto nos dice:

La familia es un fenómeno primordial ya que es el elemento fundante de la sociedad desde el inicio de la historia humana. Más concretamente, la sociedad nace cuando nace la familia (Lévi-Strauss, 1969), nace «con» ella, en el mismo momento y del mismo modo. En los inicios de la humanidad, la sociedad se forma a partir de que hace familia (se dice que la familia, en aquel momento, coincide con 
la sociedad). Al principio de la existencia temporal de una sociedad no existe el individuo aislado, sino el grupo familia: si el individuo está completamente aislado, muere. Toda sociedad asume la forma de una amplia parentela (tribu, clan o gens), y la sociedad que se va desarrollando está constituida por las relaciones entre tribus y clanes. Por tanto, en un primer sentido, la familia es primordial en cuanto que sin ella lo social no adquiere existencia histórica (2003, p. 21).

Segundo, propone que la familia da lugar a la civilización. Sobre esto afirma:

[La familia] es un fenómeno primordial porque, en el curso de la evolución humana, es la matriz fundamental del proceso de civilización (Zimmerman, 1971). Aquí primordial no significa que está en el origen de la civilización, sino que, en el contexto y en el presente, también en niveles más desarrollados y diferenciados de la evolución social, la familia es precondición de toda posible adquisición de civilización. No se alude ahora a que la familia sea el lugar (sistema social primario viviente) en que se reproduce la especie humana. Se alude a algo más esencial. Se indica que una sociedad no puede existir si no dispone de una cultura que pueda pensar y vivir de forma «familiar», y esto está más allá del horizonte meramente familiar (es lo desconocido, extraño, lo no familiar) (Horkheimer-Adorno, 1966). Aún más, la capacidad de desarrollo de cualquier sociedad consiste en saber traducir lo no-familiar en familiar (Kuhmann, 1989b). Para hacerlo, debe recorrer categorías simbólicas que tienen en la familia su arquetipo. En este sentido, la familia es primordial en cuanto que encarna una distinción (familiar/no-familiar) sin la cual el proceso de civilización no puede avanzar (Donati, 2003, p. 21).

En relación con lo anterior, la familia se postula como un escenario educativo, para la construcción de la sociedad y para la configuración de la identidad de los sujetos y de su condición como seres sociales, pues es evidente que ella transmite la carga simbólica inicial, con la que se construyen un sentido de mundo, del otro y del yo individual, para ellos mismos y para la comunidad que habitan.

\section{Familia, educadora de virtudes}

Tal como lo han mostrado algunos estudios adelantados por Ramos (1990), Jaime (2000), Barbeito (2002), Nateras (2003), Tapia (2003), Abela (2003), Rodríguez (2007), Jaime (2010), Cebotarev (2011), Aguirre (2011), Rincón (2012), Gonzáles (2015), Patiño (2014), entre otros, la familia transmite los principales códigos sociales que le permiten a los niños realizar su agenciamiento como individuos, es decir, como seres políticos, y como actores sociales.

El rol de la familia como un agente educativo, facilita su valoración como una unidad social que, por su rol natural, genera el desarrollo de esquemas sociales, como es el caso de las virtudes, centrales para la vida en comunidad de un sujeto. Sobre esto, Isaacs (2010) plantea que es posible lograr que las personas:

Desarrollen las virtudes motivadas por el amor, por saber que todo miembro de la familia tiene el deber de ayudar a los demás miembros a mejorar, porque mientras uno convive con otras intimidades en una organización natural, lo que crece o lo que se enferma es un mismo cuerpo, una misma entidad, la familia... En la familia, porque existe confianza personal en los demás y porque existe confianza en el futuro, en las potencialidades de las personas por ser únicas y valiosas, se pueden elevar las miras de los niños de tal modo que impregnan sus actos con amor, y llegan a amar el mundo apasionadamente... La familia hemos dicho es sede del desarrollo de las virtudes humanas... Cabe advertir que, sin la familia, difícilmente se podría conseguir el desarrollo de estos hábitos operativos buenos, contando con la intensidad con que se viven y con la rectitud de los motivos al vivir. (pp. 25-29).

La particularidad educativa de la familia se encuentra en que se encarga de modo peculiar del desarrollo de hábitos operativos, los cuales se transmiten mediante la vivencialidad, la ejemplaridad y la reflexividad. La vivencialidad es una acción educativa íntima que alimenta de modo permanente los esquemas con los que se entienden los fenómenos del mundo, las virtudes y las actuaciones virtuosas. La enseñanza ejemplar para Steiner es la única autoridad pedagógica honrada y estable (2011). Así, la ejemplaridad será la única didáctica honrada por la cual la familia podrá transmitir el valor, el sentido y el significado de la virtud y de las acciones virtuosas. Esto lleva a reconocer que la ruptura entre el discurso y la práctica en la enseñanza de las virtudes conduce no solo a la contradicción, sino también a la pérdida de legitimidad de lo que se intentaba enseñar.

A la enseñanza formal de las virtudes se debe sumar la vivencialidad de estas, para que los niños y jóvenes evidencien de facto que son principios reales y prácticos, que llevan a la configuración de una vida buena. Pero, a la vivencialidad y la ejemplaridad 
de las virtudes hay que añadir la reflexividad; un análisis estructurado, consciente y puntual, sobre los aspectos positivos y negativos de la ausencia y presencia de las virtudes en la cotidianidad.

La puesta en práctica de estos tres principios como elementos necesarios para la enseñanza de las virtudes, no requiere de un gran esfuerzo; precisa de la consciencia de cualquiera de los padres sobre el hecho de que sus actos, hasta los más mínimos, generan un efecto educativo permanente, requiere de la disposición y apertura al diálogo; de una escucha atenta y asertiva que "supone encuentro, comunicación que apunte hacia la posibilidad de comunión que sólo se da cuando se cree, se valora al otro, cuando emerge el respeto y la confianza" (Cussianovich , 2003, p. 88).

Empero, con respecto a esto, ¿qué virtudes se podrían enseñar? Para Isaacs (2010), esta pregunta se debe responder en función a:

1) Los rasgos estructurales de la edad en cuestión,

2) la naturaleza de cada virtud, 3) las características y posibilidades reales del joven que estamos educando, 4) las características y necesidades de la familia y de la sociedad en que vive el joven [y], 5) las preferencias y capacidades personales de padres (p. 38).

Una vez se reconocen estos elementos formales, Isaacs (2010) propone que las virtudes a enseñar deberían ser: obediencia, sinceridad y el orden hasta los siete años; fortaleza, perseverancia, laboriosidad, paciencia, responsabilidad, justicia y generosidad desde los ocho hasta los doce años; desde los trece hasta los quince años pudor, sobriedad, sencillez, sociabilidad, amistad, respeto y patriotismo; desde los dieciséis hasta los dieciocho años prudencia, flexibilidad, comprensión, lealtad, audacia, humildad y optimismo. Cada estadio de virtudes se propone en función de la capacidad de aprendizaje del niño y, de su habilidad para evidenciar en su cotidianidad el valor de estos principios de acción.

\section{Familia y educación cívica}

La familia debe enfocar sus esfuerzos cotidianos al desarrollo de un conjunto de hábitos sociales operativos loables, acciones tendientes al bien; al buen uso del libre albedrío, al decir de Tomás de Aquino (1997), acciones que lleven a la familia a descubrir el valor del bien y de la excelencia.
La socialización política intencionada (formal-planificada) o no intencionada (informal), en la que la familia es un agente clave, tiene un efecto trascendente en la constitución de la identidad de la persona, sobre todo en su formación y empoderamiento como ciudadano. Al ser el primer escenario de vida y un ámbito transaccional, el significado y el sentido que desde allí se atribuyen a la ciudadanía y a sus valores serán cruciales. Si bien, todas las instituciones sociales participan desde sus particularidades en la formación y conformación de la identidad política, la familia se destaca por su presencia continua y por su condición de red de apoyo.

Savater (1997) soporta la idea de esta socialización ciudadana en el ámbito familiar, recalcando la coincidencia de los estudiosos en atribuir a la «socialización primaria», gran importancia. Como él mismo explica:

En la familia el niño aprende -o debería aprender- aptitudes tan fundamentales como hablar, asearse, vestirse, obedecer a los mayores, proteger a los más pequeños (es decir, convivir con personas de diferentes edades), compartir alimentos y otros dones con quienes les rodean, participar en juegos colectivos respetando los reglamentos, rezar a los dioses (si la familia es religiosa), distinguir a nivel primario lo que está bien de lo que está mal según las pautas de la comunidad a la que pertenece, etc. Todo ello conforma lo que los estudiosos llaman «socialización primaria» del neófito, por la cual éste se convierte en un miembro más o menos estándar de la sociedad (1997, p. 26).

Desde el punto de vista de Nussbaum (2011), por vía del ejemplo, la familia potencia o limita la capacidad que posee libre y conscientemente un ciudadano para participar en la sociedad. Es en la intimidad del hogar, en el cuidado diligente, oportuno y respetuoso entre los miembros, donde se gesta la formación ciudadana. En palabras de Savater (2002), "una vida familiar entre personas adultas comprometidas efectiva y eficazmente en el crecimiento de los niños es indispensable para crear ciudadanos capaces de enfrentarse al poder” (p. 85).

El poder socializador de este ámbito de vida se caracteriza por que sus prácticas se orientan, de modo específico, hacia la formación de un conjunto de virtudes cívicas que inciden en la actuación ciudadana de todos sus miembros. Al ser un escenario informal, las virtudes cívicas se socializan ejemplarmente. Es la reflexión praxeológica sobre una situación o un problema privado o público, que se 
lleva a cabo en este ámbito, la que tiene un alcance formativo, ya que el insumo de la socialización será la vida misma, sobre la que se discute y piensa en familia para reconocer y aprehender los hábitos sociales prudenciales o nocivos.

Para Pereira (2004), el eje de la educación cívica es la socialización de los valores y las virtudes, que conformarán la base de la actuación ciudadana y democrática:

La vitalidad de una democracia dependerá de las medidas que se tomen para articular su educación cívica, la que deberá ser tanto en virtudes como en valores. Estas dos posibles líneas de la educación cívica conforman procesos que deben estar orientados a asegurar las condiciones operativas de la democracia, que tiene en las condiciones del diálogo su criterio normativo más fuerte. En tal sentido, tanto los valores como las virtudes operarán como posibilitantes de la democracia, por lo que la realización de los valores de libertad, igualdad, respeto activo, solidaridad y diálogo, a través del ejercicio de las virtudes cívicas de sentido crítico, solidaridad, apertura al diálogo y participación activa, propiciará la construcción de la democracia deliberativa. (p. 280).

En este sentido, se entiende que la educación cívica se dirige a la formación de hábitos virtuosos, se orienta hacia la concienciación de la responsabilidad que los ciudadanos tienen hacia ellos mismos y su comunidad de vida, pero también a una vinculación real y activa con el medio social y ambiental (Vaillant, 2008; Fernández, 2014).

Las virtudes que se edifican en la educación cívica contribuyen al crecimiento material y espiritual de las personas y la sociedad, pues forman personalidades dispuestas a la aceptación radical del otro, a acoger y reconocer la dignidad inherente que hay en toda forma de vida. Esto quiere decir que "el objetivo de la educación cívica deberá ser educar la mirada de los ciudadanos para poder reconocer la humanidad en aquellas dimensiones en que suele ser negada" (Pereira, 2004, pp. 275-276); lo cual implica, una amplia labor de la familia y con una dimensión ética, pues ella se encarga de socializar en sus miembros el sentido de lo humano, su valor y defensa.

Las prácticas reflexivas familiares, como espacios de educación cívica, han de enfocarse en el desarrollo de la solidaridad (Altarejos y Naval, 2005); el significado y el sentido en la construcción social de la ciudadanía y el desarrollo de una voca- ción de servicio; el conocimiento de los Derechos Humanos, la comprensión de los problemas sociales y la cooperación comunitaria (Naval, García, Puig y Anxo, 2011, p. 88); así como también en el desarrollo de conocimientos democráticos, el aprendizaje de las habilidades y los procesos de participación cívica y política responsable (Naval, 2003, p. 13).

En resumen, dentro de este escenario, la educación cívica se postula como un eje fundamental para la formación ciudadana (Coronel, 2012, p. 169), pues contribuye a que las personas afiancen una perspectiva humanista cívica, crítica y propositiva. Igualmente, la educación familiar cívica se debe orientar hacia el desarrollo de unas aptitudes y actitudes personales. Es decir, la formación en este ámbito debe dar cuenta de unas virtudes que orienten a la persona, dentro de la trama social, hacia la tolerancia (Guichot, 2012), el respeto, la responsabilidad, el civismo, la integridad, la autodisciplina, la compasión, la honestidad, la promoción del bien común (Marina, 2005) y la "realización del diálogo como corazón de una sociedad democrática" (Pereira, 2004, pp. 267-269).

Los aprendizajes que surgen de la educación cívica conforman un sistema de principios sociales que regulan, de manera ética, la vida de los ciudadanos. Esta regulación será humanística y no contractual, dado que el sentido de las acciones de los ciudadanos se cimienta en el compromiso que tienen hacia el Otro y no, como expone Tapia (2009), en el compromiso contractual centrado en un beneficio o un castigo instrumental que ejecuta un actor social externo.

De acuerdo con lo anterior, la educación cívica familiar, redunda en la formación de una ética ciudadana, pues las virtudes que socializa inciden en la persona, en la configuración de un sentido de su responsabilidad consigo mismo y la sociedad:

La idea ética de ciudadanía nos resulta ahora muy extraña, pero los conflictos sociales nos fuerzan a recuperarla. Todas las culturas transmiten mediante la educación un modelo de persona, un modelo de comportamiento y un modelo de sociedad. El nuestro está claro: Deseamos que nuestros conciudadanos sean libres, responsables, cooperadores, solidarios, justos y compasivos. Necesitamos que su comportamiento esté dirigido por proyectos de felicidad privada que sean compatibles, compartibles y cooperadores respecto de la felicidad de los demás. Y aspiramos a una sociedad justa, que 
mejore el bienestar y las posibilidades de cada uno de sus miembros (Marina, 2005, p. 5).

Con la ética ciudadana que resulta de la educación cívica, se adquieren un conjunto de principios prácticos que ordenan la relación del sujeto con su medio social y natural. Este tipo de ética opera como un lineamiento social que estructura los comportamientos políticos. Ésta se construye en los espacios cotidianos de la realidad familiar, mediante la continua reflexión y problematización de los distintos códigos deontológicos que hacen parte de la comunidad de vida de la que se participa, así como de los comportamientos enfocados o no a la búsqueda del bien social.

Las virtudes aprendidas en el ámbito familiar dejan de estar en el fuero interno, para convertirse las acciones sociales. En este orden de ideas, la ética ciudadana ya no es el reino exclusivo de los hábitos operativos, es también el de las habilidades sociales que facilitan la ordenación del mundo, la solución de los problemas elementales y prácticos que cada persona enfrenta en su cotidianidad.

Si la familia es un bien en sí, su práctica educativa se debe dirigir ontológicamente a la socialización de virtudes cívicas y, a la conformación de una capacidad deontológica para ser un ciudadano responsable:

La noción de "ciudadanía responsable" lleva a cuestiones relativas a la sensibilización y al conocimiento de los derechos y deberes. Asimismo está estrechamente relacionada con los valores cívicos, como la democracia y los derechos humanos, la igualdad, la participación, el asociacionismo, la cohesión social, la solidaridad, la tolerancia a la diversidad y la justicia social... Por tanto, podría decirse que, por lo general, la "ciudadanía responsable" engloba aspectos relacionados con el conocimiento y el ejercicio de los derechos y responsabilidades cívicas. Igualmente, todos los países asocian el concepto con ciertos valores estrechamente relacionados con el papel de un ciudadano responsable. Entre ellos se incluye la democracia, la dignidad humana, la libertad, el respeto por los derechos humanos, la tolerancia, la igualdad, el respeto a la ley, la justicia social, la solidaridad, la responsabilidad, la lealtad, la cooperación, la participación y el desarrollo espiritual, moral, cultural, mental y físico (Eurydice, 2005, pp. 10-13).

Así, la ciudadanía responsable se puede entender como la condición política, social y cultural con la que las personas actúan y reflexionan crítica- mente sobre ellas mismos, su comunidad local, el bien común y el contexto global; refleja la capacidad de enunciación cotidiana de los derechos y deberes, pero también, del sentir común de los miembros de su comunidad local y global. La ciudadanía responsable se explica como condición constitutiva per se, ya que es un escenario de comunicación en el que se hace posible el desarrollo de la identidad del ser humano como ser social solidario; de esquemas de pensamiento y acción que lo hacen consciente de su lugar en el mundo y de sus responsabilidades cívicas. La ciudadanía responsable es el escenario donde se vinculan e igualan, en lo político, todos los individuos, pues esta condición no depende de un rol otorgado, ni de unos beneficios que adquiridos, o de un conjunto específico de prácticas políticas, sino de los distintos mecanismos políticos y sociales por medio de los cuales se ejercen los derechos, los deberes, la libertad y la autonomía.

\section{Conclusión}

Uno de los principales aportes de la familia como institución estructuradora a la persona y la sociedad es la socialización política; en especial la educación en virtudes cívicas. Esto evidencia que es en la cotidianidad de la vida familiar donde se siembra la semilla que hace posible y real una democracia participativa y dialogante-deliberativa, ya que, a la vez que se es un buen padre y actor cívico, se es también un buen educador, un virtuoso socializador de principios democráticos que da lugar en su hogar a la concertación, el respeto, la justicia, la conversación solidaria y la escucha activa. Así, la educación cívica es "un elemento de construcción personal y social, un recurso para el conocimiento de la problemática social contemporánea y de los valores y proyectos éticos presentes, un incentivo para el reconocimiento y el ejercicio del compromiso cívico y la responsabilidad" (Mayordomo, 2008, p. 231).

Cuando se reconoce que la familia no es un transmisor pasivo, sino un actor social clave que orienta los procesos educativos hacia la búsqueda de metas propias, se abre la oportunidad para valorar la capacidad de encausar, de manera intencionada, la formación de sus miembros hacia la construcción de principios fundamentales de acción. Fundamentos "capaces de nutrir una moral voluntaria deliberante (educación de juicio) vis a vis la apertura a una educación orientada a ampliar la solidaridad y a la práctica de virtudes ciudadanas referidas a los 
derechos humanos y a la creación de sociedades de cooperación" (Osorio, 2004, p. 10).

La educación cívica familiar es una formación orgánica, es decir, una formación vital de las normas, pues se fundamenta en la capacidad que tienen los actores familiares de hacer vivos los principios, de creerlos y acogerlos como elementos esenciales para el desarrollo de su propia existencia. De acuerdo con Marina (2005), esta formación se centra en la estructuración de hábitos de vida orientados al perfeccionamiento de la persona y de competencias cívicas asumir, como diría Mayordomo, (2008) "el protagonismo plural, la colaboración social constructiva, la responsabilidad del compromiso activo" (p. 222).

En la familia, con sus prácticas cotidianas, se forma en y para una ciudadanía responsable que, como se ha señalado, es el resultado de la educación cívica y de la formación en una ética ciudadana centrada en el reconocimiento del valor del bien común y de la dignidad que posee cualquier forma de vida. Igualmente, se reconoce el valor de su participación en el involucramiento permanente y crítico de la persona en los procesos que afectan positiva o negativamente a la sociedad.

Es en la familia donde se construye el sentido definitivo y extenso de lo que define la condición de una persona como ciudadana. Los aspectos normativos y legales se adquieren después de la escuela (Castro y García, 2016; Chaparro, Gonzáles y Caso, 2016; Gomariz, Hernández, García y Parra, 2017). Es aquí, en la familia, donde inicia la formación ciudadana, la formación que será estable y duradera, la que pre-condicionará los comportamientos políticos y ciudadanos.

\section{Referencias}

Abela, J. (2003). Infancia socialización familiar y nuevas tecnologías de la comunicación. Portularia, 3(1), 243-261. Recuperado de http://hdl.handle. net/10272/156

Aguirre, V. (2011). Familia y socialización política de jóvenes en Argentina. Hologramática, 6(15), 35-61. Recuperado de http://www.cienciared.com.ar/ra/ usr/3/1173/hologramatica_n15v1pp35_61.pdf

Altarejos, F., y Naval, C. (2005). La educación cívica en una sociedad globalizada. Revista Galega do Ensino, 13(46), 842-862. Recuperado de https://dialnet. unirioja.es/servlet/articulo?codigo $=1358615$

Barbeito, R. (2002). La familia y los procesos de socialización y reproducción sociopolíticas de la ju- ventud. Revista Estudios de Juventud, 58(2), 1-11. Recuperado de http://www.injuve.es/sites/default/ files/articulo6.pdf

Castro, A., y García, R. (2016). Vínculos entre familia y escuela: visión de los maestros en formación. Magis, Revista Internacional de Investigación en Educación, 9(18), 193-208. doi: 10.11144/Javeriana. m9-18.vfev

Chaparro, A., Gonzáles, C., y Caso, J. (2016). Family and academic performance: Identifying high school student profiles. Revista Electrónica de Investigación Educativa, 18(1), 53-68. Recuperado de http:// www.scielo.org. $\mathrm{mx} /$ scielo.php?script=sci_arttext\&pid=S1607-4041201600010000 4

Coronel, F. (2012). Diálogo de saberes para la conformación de una educación cívica y ciudadanía críticas. Integra Educativa, 5(3), 157-170. Recuperado de http://www.scielo.org.bo/pdf/rieiii/v5n3/v5n3ao8. pdf

Cussiánovich, A. (2003). Protagonismo, participación y ciudadanía como componente de la educación y ejercicio de los derechos de la infancia. En Historia del pensamiento social sobre la infancia. Lima, Perú: Fondo Editorial de la Facultad de Ciencias Sociales.

D’Agostino, F. (2006). Filosofía de la Familia. Madrid, España: Instituto de Ciencias para la Familia.

De Aquino, T. (1997). De las Virtudes. Traducción de Patricia Serrano Guevara. Santiago de Chile: Universidad de los Andes.

Donati, P. (2003). Manual de sociología de la familia. Navarra, España: EUNSA.

Estupiñán, J., y Hernández, A. (2007). Lineamientos técnicos para la inclusión y atención de familias. Bogotá, Colombia: ICBF.

Eurydice. (2005). La educación para la ciudadanía en el contexto escolar europeo. Bruselas, Bélgica: Comisión Europea.

Fernández, J. (2014). Conflicto, virtud y educación. Las virtudes cívicas como respuesta al conflicto. Fragmentos de Filosofía, 12(1), 25-45. Recuperado de http://institucional.us.es/revistas/fragmentos/12/ art_2.pdf

Gomariz, M., Hernández, M., García, M., y Parra, J. (2017). Tejiendo puentes entre la escuela y la familia. El papel del profesorado. Bordón, 69(2), 41-57. doi: 10.13042/Bordon.2016.49832

Gonzáles, D. (2015). La familia como texto y contexto para la socialización política de los niños y las niñas. Khatarsis, 19(1), 99-133. doi: https://doi. org/10.25057/25005731.491

Guichot, V. (2012). Tolerancia, una virtud cívica clave en una educación para la ciudadanía activa, compleja 
e intercultural. Bordón, 64(4), 35-47. Recuperado de https://recyt.fecyt.es/index.php/BORDON/article/view/22072

Hall, S. (2016). Moral geographies of family: articulating, forming and transmitting moralities in everyday life. Social and Cultural Geography, 17(8), 10171039. doi: 10.1080/14649365.2016.1147063

Isaacs, D. (2010). La educación de las virtudes humanas y su evaluación. Navarra, España: EUNSA.

Jaime, A. (2000). Familia y socialización política. La transmisión de orientaciones ideológicas en el seno de la familia española. Reis. Revista Española de Investigaciones Sociológicas, 99(2), 71-92. doi: $10.2307 / 40184294$

Jaime, A. (2010). Familia y socialización política. La transmisión intergeneracional de las preferencias políticas. Andalucía, España: Fundación Centro de Estudios Andaluces.

Marina, A. (2005). La educación cívica. Revista Educación y Futuro, 13(1), 1-7. Recuperado de https://dialnet. unirioja.es/servlet/articulo?codigo $=2239622$

Matos, J. (2015). La Familia: The Important Ingredient for Latina/o College Student Engagement and Persistence. Equity and Excellence in Education, 48(3), 436-453. doi: 10.1080/10665684.2015.1056761

Mayordomo, A. (2008). El sentido político de la educación cívica: libertad, participación y ciudadanía. Revista Iberoamericana de Educación, 47(2), 211235. Recuperado de: https://rieoei.org/historico/ documentos/rie47a1o.htm

Muaze, M. (2016). Pensando a família no Brasil: Ganhos interpretativos a partir da micro-história. Confluenze, 8(1), 10-27. doi: 10.6092/issn.2036o967/6247

Nateras, O. (2003). Procesos de socialización política y construcción del pensamiento social en infantes y jóvenes: la ruta de la sociocognición. Polis: Investigación y Análisis Sociopolítico y Psicosocial, 2(3), 49-78. Recuperado de https://revistas-colaboracion.juridicas.unam.mx/index.php/polis/article/ view/16822/15047

Naval, C. (2003). Orígenes recientes y temas clave de la educación para la ciudadanía democrática actual. Revista de Educación, Número Extraordinario, 169-189. Recuperado de http://dadun.unav.edu/ handle/10171/21682

Naval, C., García, R., Puig, J., y Anxo, M. (2011). Ethical and Civic Education and the Social Commitment of University Students. Encounters in Theory and History of Education, 12(1), 77-91. doi: 10.24908/ eoe-ese-rse.v12io.3174

Nora, C. (2003). Familia, socialización y nueva paternidad. Revista Latinoamericana de Ciencias Sociales,
Niñez y Juventud, 1(2), 1-19. Recuperado de http:// revistaumanizales.cinde.org.co/rlcsnj/index.php/ Revista-Latinoamericana/article/view/329

Nussbaum, M. (2011). La crisis silenciosa. Signo y Pensamiento, 3o(25), 16-22. Recuperado de https:// revistas.javeriana.edu.co/index.php/signoypensamiento/article/view/2453

Organización de las Naciones Unidas. (2008). Declaración Universal de los Derechos Humanos. United Nations.

Osorio, J. (2004). Pedagogía y ética en la construcción de ciudadanía: la formación en valores en la educación comunitaria. Polis Revista Latinoamericana, 7(1), 1-11. Recuperado de https://journals.openedition.org/polis/6267

Patiño, J. (2014). Familia y procesos de socialización política en contextos del conflicto armado: posibilidades para la resignificación. Revista de Investigaciones UCM, 14(23), 32-47. doi: https://doi. org/10.22383/ri.v14i1.6

Pereira, G. (2004). Virtudes cívicas procedimentales, democracia participativa y educación ciudadana. Areté, Revista de Filosofía, 16(2), 243-281. Recuperado de http://revistas.pucp.edu.pe/index.php/ arete/article/view/106

Plaza, J., y Caro, C. (2016). La implicación de la familia en la formación ético-cívica de los jóvenes a través de las TIC. Aloma, Revista de Psicologia, Ciències de l'Educació i de l'Esport, 34(2), 97-106. Recuperado de http://www.revistaaloma.net/index.php/ aloma/article/view/291/202

Ramos, R. (1990). La familia como agente de socialización política. Revista Interuniversitaria de Formación del Profesorado, 9(1), 85-99. Recuperado de https://dialnet.unirioja.es/servlet/articulo?codi$\mathrm{go}=117723$

Rincón, M. (2012). La familia como escenario de socialización para la convivencia ciudadana. Revista Eleuthera, 7(1), 116-132. Recuperado de http://eleuthera.ucaldas.edu.co/downloads/Eleuthera7_8.pdf

Rodríguez, A. (2007). Principales modelos de socialización familiar. Foro de Educación, 9(1), 91-97. Recuperado de https://www.forodeeducacion.com/ojs/ index.php/fde/article/view/184/141

Rodríguez, S. (2013). Familia y sociedad: totalidad y relación. Estudios Sobre Educación, 25(1), 7-10. Recuperado de https://www.unav.edu/publicaciones/revistas/index.php/estudios-sobre-educacion/ article/view/1877

Savater, F. (1997). El valor de educar. Barcelona, España: Ariel.

Savater, F. (2002). Ética y ciudadanía. Madrid, España: Montesinos. 
Schrecker, P. (1978). La familia como institución transmisora de la tradición. En La familia. Barcelona, España: Península.

Steiner, G. (2004). Lecciones de los maestros. Madrid, España: Siruela.

Tapia, E. (2003). Socialización política y educación cívica en los niños. México D.F., México: Instituto Mora.

Tapia, E. (2009). Educación cívica y la construcción de la ciudadanía. Ánfora, 16(27), 87-96. doi: 10.30854/ anf.v16.n27.2009.119

Vaillant, D. (2008). Educación, socialización y formación de valores cívicos. Chile: PNUD.
Valdés, Á., Carlos, E., Quintana, J., y Madrid, E. (2016). Relación entre funcionamiento familiar, emociones morales y violencia entre estudiantes de primaria. Revista Mexicana de Investigación Educativa, 21(71), 1093-1110. Recuperado de http://www. scielo.org.mx/scielo.php?script=sci_arttext\&pi$\mathrm{d}=\mathrm{S} 1405-66662016000401093$

Zuluaga, J. (2004). La familia como escenario para la construcción de la ciudadanía: una perspectiva desde la socialización de la niñez. Revista Latinoamericana de Ciencias Sociales, Niñez y Juventud, 2(1). Recuperado de http://revistaumanizales.cinde.org.co/rlcsnj/index.php/Revista-Latinoamericana/article/view/327 
